\title{
For sympathectomy, age is just a number
}

\author{
Thomas Ng, MD, and Steven Milman, MD
}

\author{
From the Department of Surgery, The Warren Alpert Medical School of Brown University, Providence, RI \\ Disclosures: Authors have nothing to disclose with regard to commercial support. \\ Received for publication May 27, 2018; revisions received May 27, 2018; accepted for publication May 30, 2018; \\ available ahead of print July 26, 2018. \\ Address for reprints: Thomas Ng, MD, 2 Dudley St, Suite 470, Providence, RI 02905 (E-mail: tng@ usasurg.org) \\ J Thorac Cardiovasc Surg 2018; $156: 1757-8$ \\ $0022-5223 / \$ 36.00$ \\ Copyright (C) 2018 by The American Association for Thoracic Surgery \\ https://doi.org/10.1016/j.jtcvs.2018.05.113
}

Idiopathic or primary hyperhidrosis affects $1 \%$ to $3 \%$ of the population. ${ }^{1}$ Although idiopathic or primary hyperhidrosis may be considered a "cosmetic" disease in that it does not affect an individual's survival, it certainly can have a significant effect on the quality of life of individuals who have it. The majority of patients who undergo a sympathectomy tend to be adolescents or young adults, which is understandable, because hyperhidrosis may worsen during and after puberty, and the added requirement of networking to obtain higher education or job opportunities may place additional stress on presentation and body image. Historically, the literature would indicate that the ideal patient for sympathectomy is aged less than 25 years. ${ }^{1}$ However, few data have been devoted to evaluating the outcomes of sympathectomy in older patients. The current study by Leiderman and colleagues, ${ }^{2}$ published in this month's issue of the Journal, aims to fill this knowledge gap.

Leiderman and colleagues ${ }^{2}$ reviewed their experience with 1633 patients who underwent bilateral video-assisted thoracic surgery sympathectomy over a 15-year period, from 2000 to 2014 . For the entire study population, the investigators found improvement in sweating in $97 \%$ of the patients, with $90 \%$ reporting "great" improvement. Compensatory hyperhidrosis was seen in $70 \%$, with $5 \%$ being severe. Overall, "betterment" of quality of life was reported in $95 \%$ of the patients. By multivariable logistic regression, older age and lower body mass index were associated with clinical improvement at the primary site of sweating; combined resection of ganglia level 2 and 3 and higher body mass index were associated compensatory hyperhidrosis. Leiderman and colleagues ${ }^{2}$ stated that the chance of having no improvement in sweating after sympathectomy decreased by $12 \%$ for every year of age, and that age was not associated with compensatory hyperhidrosis, thus concluding that sympathectomy should be offered to older patients with hyperhidrosis.

Several study limitations must be pointed out. First, the long duration and retrospective nature of the study lead to poor control of important variables, such as the extent of workup to exclude secondary hyperhidrosis, the extent of the initial medical therapy, and the operative technique.

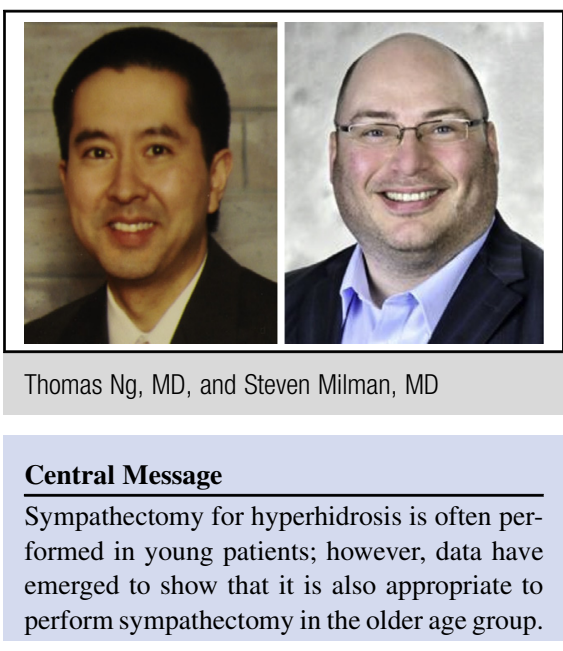

See Article page 1748 .

Both the use of initial medical therapy and the operative technique significantly changed over time. Second, the methodology used to assess quality of life may be viewed as problematic. The postoperative questionnaire was administered at 1 month; therefore, long-term data are unavailable. The questionnaires administered preoperatively and postoperatively were different, with the latter asking the patients about their perception of improvement. Having the patients fill out the same quality of life questionnaire before and after sympathectomy and calculating a change in the parameters may have been a more objective measure of outcome. Another point to keep in mind is that older individuals may have lower expectations or be more nuanced in their assessment, and therefore may be more likely to report a decrease in sweating even if it is small.

This study by Leiderman and colleagues ${ }^{2}$ shows that it is appropriate to consider sympathectomy for hyperhidrosis in older patients; however, the value of this article also may be in showing us the shift in the culture of outcomes research. Most traditional articles, especially those regarding malignant disease, have focused on younger populations and overall survival. We are seeing an aging population, though, and with advances in cancer therapy, malignant disease is being turned into a chronic condition. It therefore behooves us, in our research, to study groups that better reflect our patient population and to start focusing on the morbidity of our interventions and quality of life as much as on mortality. The authors are to be commended for this perspective. 


\section{References}

1. Cerfolio RJ, De Campos JR, Bryant AS, Connery CP, Miller DL, DeCamp MM, et al. The Society of Thoracic Surgeons expert consensus for the surgical treatment of hyperhidrosis. Ann Thorac Surg. 2011;91:1642-8.
2. Leiderman DBD, de Campos JRM, Kauffman P, Tedde ML, Yazbek G, Teivelis MP, et al. The relation between age and outcomes of thoracic sympathectomy for hyperhidrosis: the older the better. J Thorac Cardiovasc Surg. 2018;156:1748-56. 